\title{
Signaling Molecules of Human Skin Cells as the Targets for Injection Cosmetology
}

\author{
Zarema I Gazitaeva' \\ Anna O Drobintseva $\mathbb{1}^{2,3}$ \\ Aleksei Y Prokopov ${ }^{4}$ \\ Anna N Sidorina ${ }^{2}$ \\ Daria O Leonteva ${ }^{3}$ \\ Igor M Kvetnoy ${ }^{3,5,6}$ \\ 'Department of Medical Support Institute \\ of Beauty Fiji, Moscow, Russian \\ Federation; ${ }^{2}$ Department of Medical \\ Biology, Saint-Petersburg State Pediatric \\ Medical University, Saint Petersburg, \\ Russian Federation; ${ }^{3}$ Center for \\ Molecular Biomedicine, Saint-Petersburg \\ State Research Institute of \\ Phthisiopulmonology, Saint Petersburg, \\ Russian Federation; ${ }^{4}$ ROS-Chimia \\ Corporation, Ekaterinburg, Russian \\ Federation; ${ }^{5}$ Department of Pathology, \\ Saint Petersburg State University, Saint \\ Petersburg, Russian Federation; \\ ${ }^{6}$ Department of Cell Biology and \\ Pathology, Saint-Petersburg Institute of \\ Bioregulation and Gerontology, Saint \\ Petersburg, Russian Federation
}

\begin{abstract}
Introduction: Skin aging is a natural process that cannot be stopped. However, there are many ways to help attenuate premature aging of the skin and reduce the signs that have already appeared. One of them is the subcutaneous administration of preparations containing a combination of hyaluronic acid, active amino acids, and peptides providing an anti-aging clinical effect. The purpose of this research is to study in vitro new signaling molecules with the anti-aging effects and influence of hyaluronic acid fillers on its expression.
\end{abstract}

Methods: The study was conducted using cell cultures of human facial skin: 1) mixed culture of human facial skin keratinocytes and fibroblasts, and 2) culture of human facial skin keratinocytes enriched with Merkel cells. Immunocytochemistry, confocal microscopy and Western blot were used to identify markers of aging.

Results: HA-Y and HA-S activated the expression of Klotho in the case of aging mixed culture of human skin keratinocytes and Merkel cells. The increase in expression of MTH-1 with aging of cultures provides evidence of activating defense mechanisms against reactive oxygen species that are accumulating with aging, under the action of HA-S and HA-Y. There was a statistically valid increase in the area of expression of melatonin receptor $1 \mathrm{~A}$ and $1 \mathrm{~B}$ markers when adding both HA-S and HA-Y to cultured cells.

Conclusion: This investigation showed that the studied fillers have biological effects, testifying the stimulation of reparative processes in the skin under their control.

Keywords: skin aging, hyaluronic acid, active amino acids, collagen, reparative processes, klotho, clock, Merkel cells

\section{Introduction}

Numerous cosmetic products appear on the market every year, but only a few have been created on the basis of the latest achievements in science and technology, using knowledge in physiology and biochemistry of skin and complex intercellular interactions. The main components of today's innovative cosmetics are plant extracts and ingredients obtained by using biotechnology and bioengineering methods, including peptides, peptide complexes, oligo- and polysaccharides, vitamins, enzymes, and antioxidants. ${ }^{1}$ The innovation of Novacutan ${ }^{\circledR}$ medical products consists in the technology solution called HOPAAB (Hyaluronat Optically Pure Amino Acid Bond), a combination of hyaluronic acid, active amino acids, and peptides. HOPAAB is a complex of optically pure amino acids built into the network structure of hyaluronic acid, which activates the expression of signaling molecules leading to changes in metabolism, functions and development of damaged cells. Hyaluronic acid is a unique biopolymer having hydrophilic properties and moisturizing effects on the skin.
Correspondence: Anna O Drobintseva Department of Medical Biology, SaintPetersburg State Pediatric Medical University, Litovskaya Str., 2, Saint Petersburg, 194100, Russian Federation $\mathrm{Tel}+79811853607$

Fax +7 495 694-20-45

Email anna.drobintseva@gmail.com 
Geriatricians anticipate life extension in Russia from current 73.5 years to 80.1 years by 2030. The loss of physical attractiveness often leads to a decrease in selfesteem and quality of life, especially at the age of 35-60 years, and this group of people are most interested in improving their appearance. ${ }^{2} \mathrm{HA}-\mathrm{Y}$ and HA-S are administered for different age groups: while HA-Y can be utilized for younger patients of $25+$ years to fight against dark under-eye circles and improve skin hydration, HA-S, having a double concentration of amino acids, fights against wrinkles and provides a lifting effect, and can be used for patients of 35 to 65 years old.

Investigation of HA-Y and HA-S preparation effects on various environmental oxidative stress ageing markers (blue light, UVA/UVB, Urban Dust, and IR exposures) were conducted in an independent laboratory in Belgium (StratiCELL SA). The oxidative stress studies aiming to highlight possible protective effects through different challenging conditions in two models, ex-vivo human skin explants and in vitro monolayer cultures of normal human dermal fibroblasts (NHDF). The protective effects of the test products were evaluated against an increase in Cyclobutene Pyrimidine Dimers (CPDs) abundance within epidermal section of ex-vivo skin explants after UVA/ UVB radiation; effects of blue light on gene expression from NHDFs fibroblasts; effects of pollutants (Urban dust, $\mathrm{UbD}$ ) on gene expression in NHDFs fibroblasts; and, an increase in Reactive Oxygen Species (ROS) production by NHDFs fibroblasts after infrared-A radiation. Some interesting findings emerge during this investigation, for example, beneficial effects against blue light induced oxidative stress of HA fillers; up-regulation of DNA damage-inducible transcript 3 protein (DDIT-3) in the urban dust tests, etc.

The HA fillers contain active ingredients clinically effectiveness of which are well documented. For example, free amino acids contribute to the improvement of the skin water and protein balance, maintaining the skin's elasticity. Peptides have a revitalizing effect on the damaged skin, while the introduction of moisturizing components produces the expected moisturizing effect and results in skin tightening and improving the complexion. ${ }^{3}$

Peptides provide a remarkable example of multifunctional agents, which are widely used by the world's leading manufacturers of cosmetics. Peptides not only effectively fight against age-related changes in the skin but also provide protection against damaging factors. ${ }^{4}$
The purpose of this research is to study the effects of one injectable HA-based products (packed in a syringe as a sterile ready-to-use solution) on the expression of signaling molecules potential geroprotectors in facial skin cell cultures.

\section{Materials and Methods}

The study was conducted using cell cultures of human facial skin: 1 - mixed culture of human facial skin keratinocytes and fibroblasts, and 2 - culture of human facial skin keratinocytes enriched with Merkel cells. The second model object was selected because of its unique subpopulation. Merkel cells are neuroendocrine skin cells localized in the outer layers of hair follicles, in the epidermal spinous and basal layers, and in mucous membranes. The endocrine functions of Merkel cells are associated with various growth factors and neuropeptides localized there. As Merkel cells contain bombesin, vasoactive intestinal polypeptides, nerve growth factors, and metenkephaline, they have a trophic effect on growing skin appendages and nerves. ${ }^{7}$

Cell cultures were extracted from adult skin samples obtained during circular face lift operations (women born in 1973, 1971, and 1967). The Ethics Committee of the Saint-Petersburg Institute of Bioregulation and Gerontology approved all aspects of the study design, and written consent was received from all participants. The study was conducted in accordance with the Declaration of Helsinki Ethical Principles. To evaluate the expression of age markers, the culturing was carried out to passage 2 ("young" cells) and passage 6 ("old" cells) for mixed culture of keratinocytes and fibroblasts, and to passage 1 and passage 3 for culture enriched with Merkel cells. These checkpoints were selected because of the difficulty of maintaining Merkel cells and keratinocytes in culture and their limited proliferation potential.

Hyaluronic Acid filler Y (HA-Y) (2.3\% aqueous solution of 1.4 MDa non-cross-linked HA containing Arginine, Valine, Threonine, Phenylalanine, Glycine, Glutamic acid, Proline, and Methionine); and Hyaluronic Acid filler $\mathrm{S}$ (HA-S) $(2.3 \%$ aqueous solution of $1.4 \mathrm{MDa}$ non-crosslinked HA containing Arginine, Valine, Threonine, Phenylalanine, Glycine, Glutamic acid, Proline, and Methionine). The agent was administered starting from passage 1. In order to determine the optimal concentration for analysis of the HA filler, a preliminary dose-finding experiment was performed. According to cell viability 
measurement $3 \%$ of HA filler were chosen from five concentrations. The agent was administered at each passage.

The study comprised 3 groups:

1 - control group (medium $=$ hyaluronic acid); 2 administration of HA-Y; 3 - administration of HA-S.

\section{Immunocytochemical Analysis}

The following primary monoclonal antibodies were used for an immunocytochemical investigation: type I collagen (1:150, Abcam), elastin (1:50, Abcam), AP-1 (1:200, Sigma), Klotho (1:250, Abcam), MTH-1 (1:1000, Abcam), melatonin receptor $1 \mathrm{~A}$ (1:200, Abcam), melatonin receptor $1 \mathrm{~B}$ (1:100, Abcam), clock (1:100, Abcam), cytokeratin 20 (1:150, Dako). The secondary antibodies conjugated with Alexa Fluor 488 and 647 (1:1000, Abcam) were used for immunofluorescent microscopy. Confocal microscopy was carried out using an Olympus FV 1000 microscope (Japan).

Since the investigation was concerned with unique dermal Merkel cells, to confirm the existence of a subpopulation of these cells in the culture, we used a special marker cytokeratin-20. We applied the method of double labeling using antibodies to cytokeratin-20 (green fluorescence) and antibodies to Klotho (red fluorescence). The area of expression was calculated in relation to Merkel cells that were immunopositive to CK-20 cells.

\section{Western Blot Analysis}

All cells were lysed on ice with the subcellular protein Fractional Kit containing protease inhibitor cocktail (Thermo Fisher, USA). Protein lysates were separated by sodium dodecyl sulfate-polyacrylamide gel electrophoresis (SDS-PAGE), and transferred to nitrocellulose membranes (Invitrogen, USA).

The membrane is blocked with commercial set iBindTM Solution Kit (Invitrogen, USA) and then incubated with primary and secondary antibodies using the device iBind (Invitrogen, USA) for 2 hours. Antibodies were used in this study included rabbit anti-Klotho (1:1000, Abcam), rabbit anti-AP-1 (1:500, Sigma), rabbit anti- $\beta$-actin (1:5000, Abcam) and horseradish peroxidase (HRP-) conjugated secondary antibody (anti-rabbit IgG; 1:10000; Invitrogen). The intensity of each band was quantified by iBright Analysis Software (Invitrogen).

The statistical analysis of experimental data included the computation of the arithmetic average, standard deviation, and confidence intervals for each sampling. The comparison of the groups was done using the MannWhitney U-criterion.

\section{Results of in vitro Investigation of HA Based Filler Geroprotective Properties}

\section{The Results of the Investigation Carried Out on Human Skin Culture Enriched with Merkel Cells}

With a longer time of cultivating, the form of cells labeled with CK-20 changed from rounded to more flattened and dendritic in 3-8 days. In 8 days after the start of cultivating, Merkel cells did not show any signs of nuclear disorganization peculiar to apoptosis.

The statistical processing of morphometric analysis data for the expression of Klotho marker in the control group (with administered hyaluronic acid) did not show any differences between passages 1 and 3. With the administration of HA-S at passage 1 the area of expression was $5.28 \pm 0.74 \%$, whereas at passage 3 it almost doubled to $9.23 \pm 0.89 \%$. A similar picture was observed in the group with administration of HA-Y, where the expression of Klotho with aging increased by 2 times. Thus, HA-Y and HA-S activated the expression of Klotho in the case of aging mixed culture of human skin keratinocytes and Merkel cells.

Another signaling molecule that prevents aging is melatonin (MT). An important feature of melatonin is its ability to control cell division. MT inhibits cell mitosis by delaying the division metaphase.

We also studied the expression of melatonin receptors. There was a statistically valid increase in the area of expression of melatonin receptor $1 \mathrm{~A}$ marker when adding both HA-S and HA-Y to cultured cells.

Below are microphotographs (Figure 1) showing the expression of melatonin receptor $1 \mathrm{~A}$ marker. It should be noted that red fluorescence was observed not only in Merkel cells but in keratinocytes as well, although it was more expressed in Merkel cells, which had a trophic action on growing skin appendages and nerves.

The analysis of data regarding the relative area of expression of melatonin receptors $1 \mathrm{~B}$ has shown that at passage 1 there were no statistically valid differences between the control group and the HA-Y/HA-S administered group, whereas at passage 3 the relative expression area of the receptor MelR1B marker increased by $4 \%$ with 


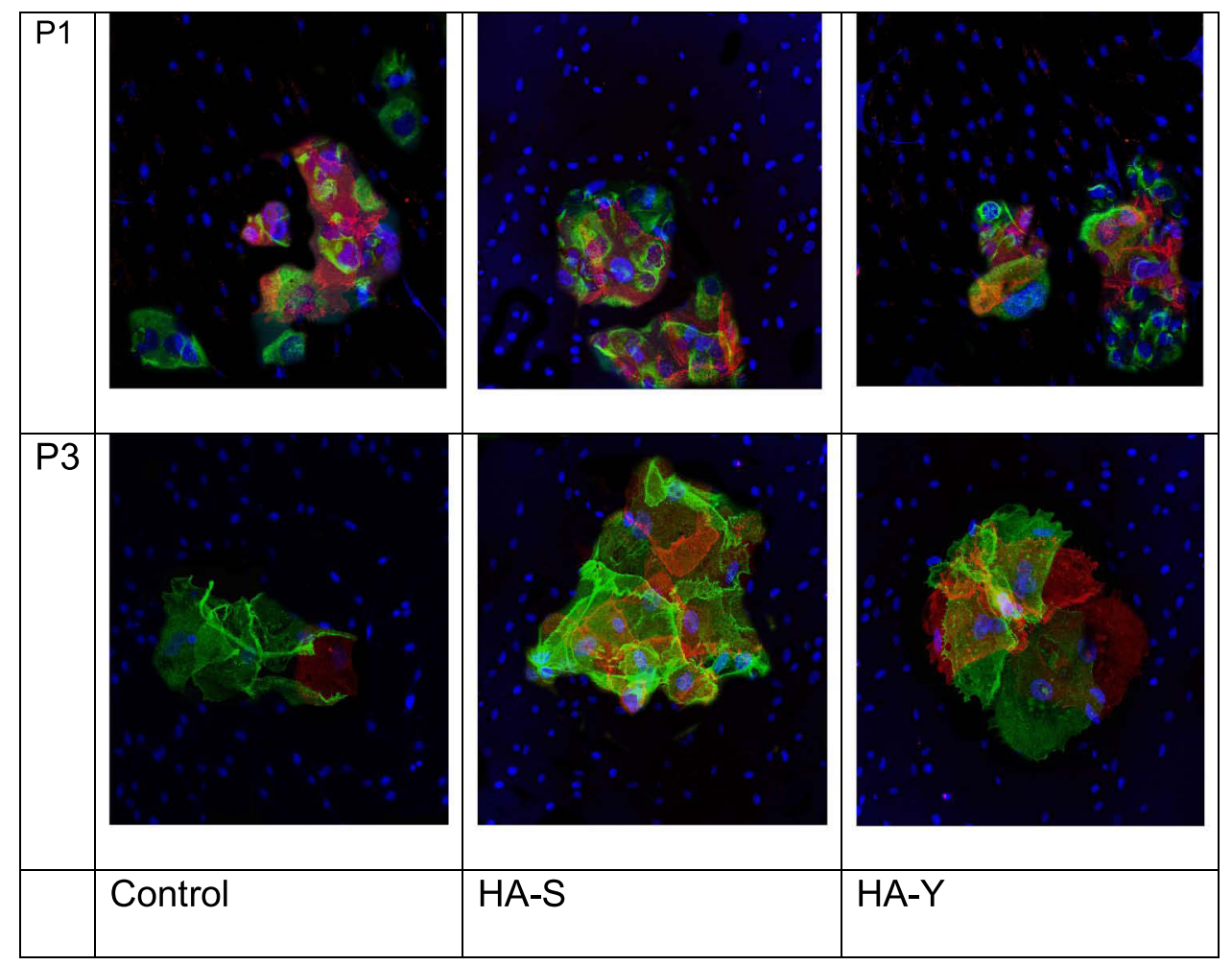

Figure I Expression of melatonin receptor IA in keratinocytes enriched with Merkel cell culture at passage I and 3; DAPI stained nuclei; expression of cytokeratin-20 green fluorescence; expression of the protein under study - red fluorescence; magnification $\times 40$.

administration of HA-S and by $11 \%$ with administration of HA-Y.

\section{The Results of the Investigation Carried Out on Mixed Culture of Human Fibroblasts and Keratinocytes}

The next stage of investigation was testing of geroprotective properties of the new HA-Y and HA-S medications on a mixed culture of human fibroblasts and keratinocytes. The following immunohistochemical markers were selected for the investigation: Elastin, Klotho, MTH1, AP-1, clock (data in Table 1).

Antibodies to elastin were used to investigate the cell synthetic activity. Changes in elastic fibers are most characteristic for photoaged skin.

In the normal condition (control group), with skin culture aging, there was observed a decrease in the expression of elastin by human skin fibroblasts. In the groups with administration of HA-Y and HA-S a similar picture was observed, yet the expression of the Elastin marker in "younger" cell cultures with HA-Y and HA-S exceeded the expression in control samples by 0.17 and 0.26 times, respectively. There was also a statistically valid increase in the area of Elastin expression for the samples of "older" cell cultures with HA-Y and HA-S by 0.14 and 0.19 times, respectively, as compared with control samples.

The action of HA-Y and $\mathrm{S}$ in the mixed culture of human skin fibroblasts and keratinocytes triggers the synthetic activity of cells, which is evidenced by higher expression of elastin both at passage 3 and passage 6 (Figure 2).

AP-1 or activator protein-1 is a transcription factor that regulates gene expression in response to various stimuli. Such stimuli include cytokines, growth factors, stress, and various bacterial and viral infections. ${ }^{16}$ This study demonstrated a statistically valid increase in the average expression area of AP-1 in control samples of "older" cell cultures as compared to "younger" cell cultures; whereas in the experimental groups, with culture aging there was observed a decrease in the expression of AP-1 marker by 0.2 times in case of HA-Y and by 0.24 times in case of HA-S, as compared with the control group.

There was also a statistically valid increase in the area of MTH-1 expression for the samples of "older" cell cultures with HA-Y and HA-S by 0.10 and 0.11 times, respectively, as compared with control samples. The higher expression of MTH-1 in "older" cultures provides 
Table I The Influence of HA Fillers (HA-Y and HA-S) on the Area of Signaling Molecules Expression in Mixed Culture of Keratinocytes and Fibroblasts

\begin{tabular}{|c|c|c|c|}
\hline \multirow[t]{2}{*}{ Marker } & \multirow[t]{2}{*}{ Group } & \multicolumn{2}{|l|}{ Area of Expression (\%) } \\
\hline & & Passage 3 (Mean \pm SD) & Passage 6 (Mean \pm SD) \\
\hline \multirow[t]{3}{*}{ AP-I } & Control & $15.54 \pm 1.18$ & $21.86 \pm 1.82$ \\
\hline & HA-Y & $16.02 \pm 1.45$ & $16.94 \pm 1.32$ \\
\hline & HA-S & $15.86 \pm 1.12$ & $16.52 \pm 1.04$ \\
\hline \multirow[t]{3}{*}{ Klotho } & Control & $1.12 \pm 0.16$ & $1.36 \pm 0.09$ \\
\hline & HA-Y & $1.34 \pm 0.08$ & $5.05 \pm 0.37$ \\
\hline & HA-S & $1.47 \pm 0.12$ & $4.98 \pm 0.41$ \\
\hline \multirow[t]{3}{*}{ Clock } & Control & $11.41 \pm 1.13$ & $7.25 \pm 0.83$ \\
\hline & HA-Y & $13.43 \pm 0.98$ & $9.24 \pm 1.19$ \\
\hline & HA-S & $15.28 \pm 1.21$ & $10.78 \pm 1.07$ \\
\hline \multirow[t]{3}{*}{ Elastin } & Control & $48.63 \pm 4.54$ & $26.05 \pm 3.62$ \\
\hline & HA-Y & $57.32 \pm 3.91$ & $35.8 \pm 3.7$ \\
\hline & HA-S & $55.39 \pm 4.65$ & $39.5 I \pm 3.58$ \\
\hline \multirow[t]{3}{*}{ MTH-I } & Control & $20.74 \pm 2.4$ & $|4.53 \pm 2.8|$ \\
\hline & HA-Y & $21.28 \pm 2.72$ & $19.87 \pm 2.69$ \\
\hline & HA-S & $22.06 \pm 2.92$ & $19.43 \pm 2.66$ \\
\hline
\end{tabular}

evidence of activating defense mechanisms against reactive oxygen species that are accumulating with aging, under the action of HA-Y and HA-S. This is what most studies on MTH-1 deal with. Yoshimura et $\mathrm{al}^{19}$ have demonstrated on human fibroblast culture that MTH-1 can restore DNA damages caused by oxidation with

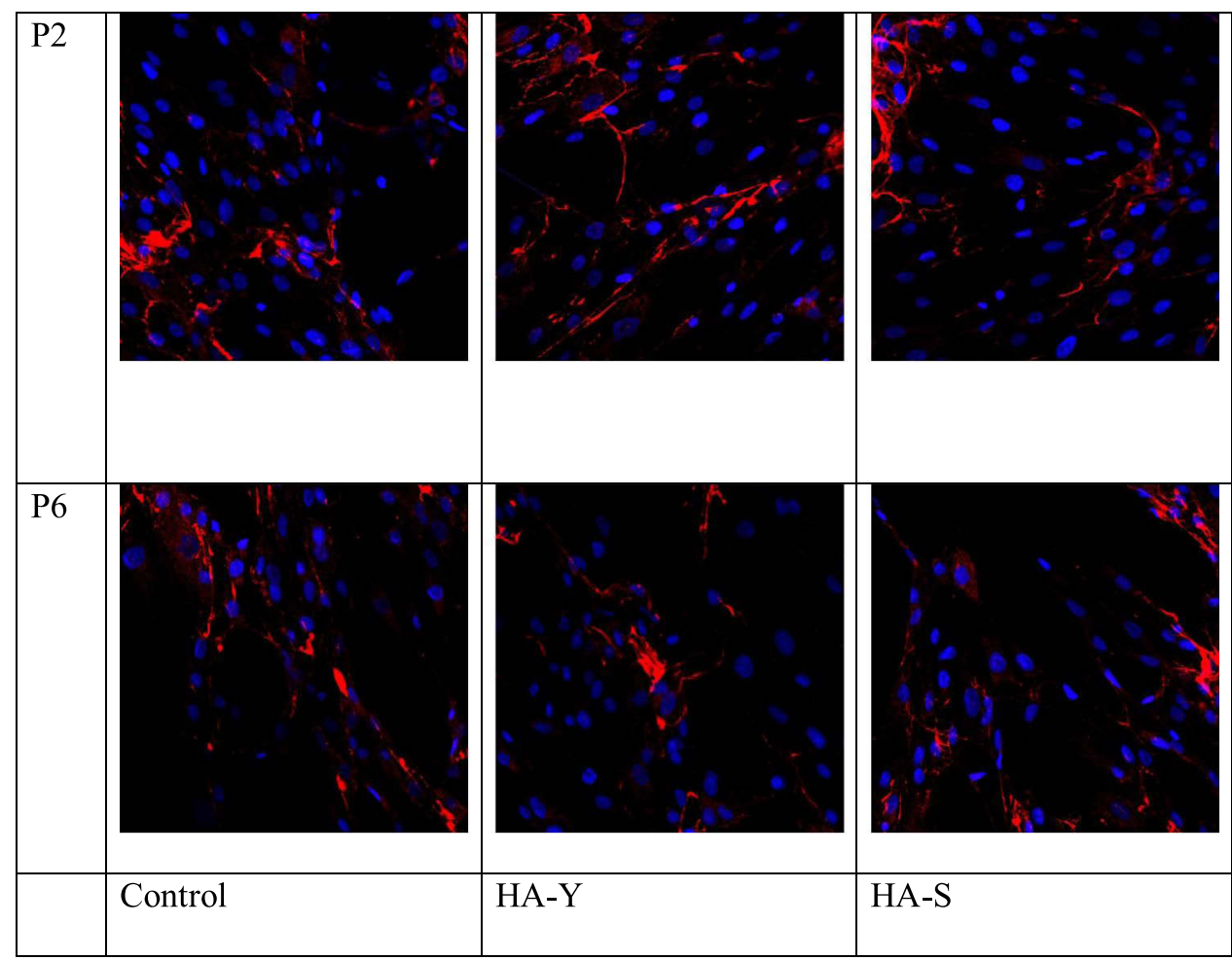

Figure 2 Immunofluorescent analysis of Elastin marker expression in cell culture. Cell nuclei were counterstained with Hoechst. Magnification x40. 
peroxide (H2O2). The analysis of the relative Klotho expression area in mixed culture of human fibroblast and keratinocytes has revealed a statistically valid increase in the average area of Klotho expression in "older" cell cultures with HA-Y and HA-S by 0.72 and 0.73 times, respectively, as compared with control samples. These results were confirmed with Western blot analysis. The protein expression had the same mode as shown in Figure 3. The higher expression of Klotho under the action of HA-Y and HA-S provides evidence of increased resistance to oxidative stress, which is especially important for prophylaxis of aging and under UV exposure.

By conducting an immunocytochemical analysis, the CLOCK marker was visualized in fibroblast cytoplasm. There was a statistically valid decrease in relative CLOCK expression area in control samples in aging fibroblast cell culture, whereas in the case of adding HA-Y and HA-S to cell cultures, the difference between "younger" and "older" cultures leveled out. It was also found that in cultivating fibroblasts in the medium with HA-Y, there was an increase in the relative expression area at passage 6 by 1.9 times as compared with the control group. HA-S had the following effect on fibroblast culture: its administration increased the CLOCK expression by 1.5 times at passage 3 , and by 2 times at passage 6 .

\section{Discussion}

According to the study entitled "Counterfeit products in aesthetic medicine: A view of the doctor and consumer" (2019, conducted with participation of Merz Russia and
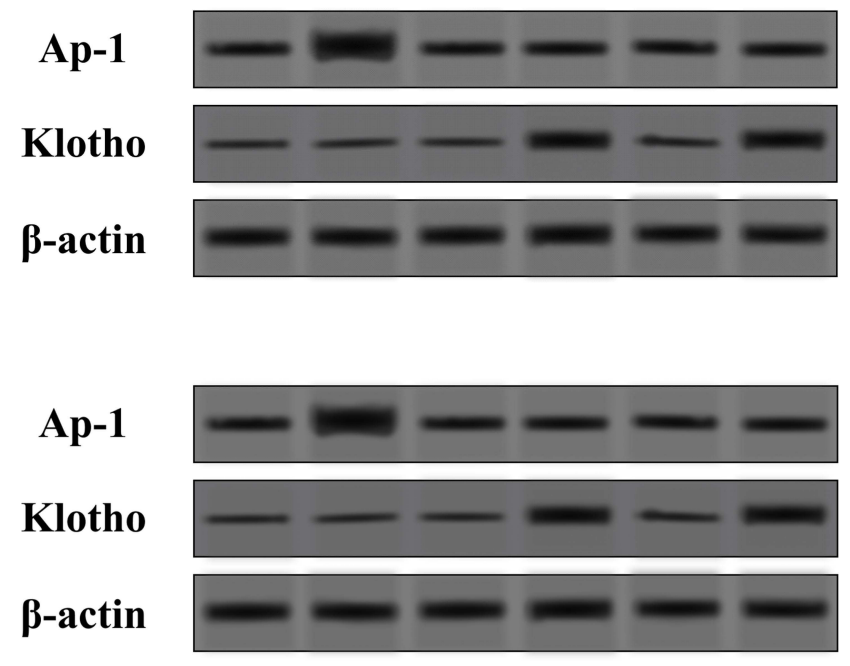

Figure 3 Expression levels of AP-I and klotho proteins in the mixed culture of human fibroblasts and keratinocytes in the HA-S and HA-Y treated group and the control (Con) group tested by Western blot.
BrandMonitor), the trafficking of counterfeit goods is estimated by experts to 200 million US dollars. Counterfeit products not only mean losses for manufacturers but can cause irreparable damage to health. Therefore, launching a new Russian injection revitalizer tested in leading laboratories in Belgium and Russia is of vital importance. ${ }^{6}$

The below listed characteristics of markers investigated in the present work include geroprotective effects, their influence on dermal homeostasis and alteration of their expression induced by HA-Y and HA-S preparations.

The administration of HA-fillers increases the expression of the Klotho protein in culture of Merkel cells and keratinocytes. Since this protein increases the resistance to oxidative stress and regulates the cell cycle, its higher expression is a favorable factor slowing down the aging process in cell culture. Klotho is a transmembrane protein; its overexpression increases the lifetime of cells. The absence of this protein in knockout mice causes their accelerated aging. ${ }^{8}$ Most works describing the functions of Klotho investigate the role of this protein in mechanisms of suppressing various age-related pathologies of the neural, vascular, and nephritic systems. ${ }^{9,10}$ Regarding its effect on aging of fibroblasts, in vitro experiments have demonstrated that in case of DNA damage Klotho contributes to the retardation of cell aging in primary human fibroblast cells MRC-5 through the apoptotic pathway (p53/21-dependent). ${ }^{11}$ The loss of Klotho activity in primary fibroblast cells MRC-5 leads to the phenotype of premature aging, which depends on the $\mathrm{p} 53$ protein stimulating the subsequent p-53-dependent CDK factor p21. This result points to the involvement of Klotho in inhibiting the aging process through the $\mathrm{p} 53 / \mathrm{p} 21$-sensitive pathway. It follows from the above that the Klotho expression activation under the action of HA-Y and HA-S can protect cells from premature aging.

It has been demonstrated that HA-Y and HA-S have a positive effect on expression of receptors to melatonin of $1 \mathrm{~A}$ and $1 \mathrm{~B}$ types in culture of Merkel cells and keratinocytes. HA-Y stimulated the expression of receptor to melatonin 1B in aging culture much more than HA-S. Melatonin is a potent antioxidant. The direct antioxidant effect of melatonin is realized through inactivation of free radicals that are formed during cell activities. ${ }^{12}$ Furthermore, by stimulating the synthesis of cell antioxidant enzymes, this hormone protects the nuclear DNA from free-radical oxidation. ${ }^{13}$

Several studies on laboratory animals have shown that melatonin has an antineoplastic, oncostatic action. In an 
experiment, the activation of the pineal gland function or the exogenous administration of melatonin led to a lower tumor occurrence rate, while pinealectomy had a stimulating effect on tumor growth. ${ }^{14}$

Like all other hormones, melatonin produces its effect through special receptors of 2 types: melatonin receptors 1A (MelR1A) and melatonin receptors 1B (MelR1B). MelR1A and MelR1B have been found and fully characterized in the retina, suprachiasmatic nucleus, myometrium, placenta, granulosa and corpus luteum cells, immune cells, gastrointestinal tract, and skin. ${ }^{15}$

The action of HA-Y and HA-S in mixed culture of human skin cells triggers the synthetic activity of cells, which is evidenced by higher expression of elastin in both "younger" and "older" cultures with administration of these medications into the medium. Elastosis refers to degenerative changes in the dermal tissue with increased deposition of elastin material. An obvious sign of this is elastosis - ie appearance of assemblies of amorphous elastin material. Apart from sun rays, elastin fibers can be affected by changes in the functioning of neural, endocrine and immune systems, and in metabolism. Thus, female sex hormones (estrogens) contribute to maintaining skin firmness and elasticity, stimulating the synthesis by fibroblasts of collagen, elastin fibers, and hyaluronic acid. An abrupt decrease in the level of estrogens in females during menopause can result in skin dehydration and formation of wrinkles. At the same time, with aging, there occurs an increase in the production of anti-inflammatory cytokines, which may cause inflammatory changes followed by degradation of collagen, elastin, and other skin elements.

The AP-1 expression marker increased with aging, whereas under the influence of HA-Y and HA-S it decreased to the level of "younger" culture. AP-1 can regulate the expression and function of cell cycle regulators, such as cyclin D1, cyclin A, cyclin E, p53, p21Cip1, p16Ink4a, and p19ARF, thereby playing an important role in cell proliferation processes. ${ }^{17}$

It has been found that AP-1 is involved in processes of decreasing the synthesis of collagen type I during photoaging. It happens as follows: the sun's UV radiation generates reactive oxygen species (ROS), which activate the growth factor and receptors of cytokines on skin fibroblasts. Activated receptors stimulate p38 and JNK; MAPK signaling cascade members and c-Fos and c-Jun then combine to form AP-1 which stimulates matrix metalloproteinase (MMP) transcription (MMP). ${ }^{18}$
The higher expression of MTH-1 in "older" cultures provides evidence of activating defense mechanisms against reactive oxygen species that are accumulating with aging, under the action of HA-S and HA-Y.

Currently, there are few studies that examine MTH-1 as an aging marker. Since the function of this enzyme is hydrolysis of oxidated purines, it is directly involved in aging regulation via reactive oxygen species (ROS). This is what most studies on MTH-1 deal with. Yoshimura et $\mathrm{al}^{19}$ have demonstrated on human fibroblast culture that MTH-1 can restore DNA damages caused by oxidation with peroxide ( $\mathrm{H} 2 \mathrm{O} 2)$.

The Clock marker expression was higher in a mixed culture of fibroblasts and keratinocytes when adding HA$\mathrm{Y}$ to the medium. This marker regulates cell metabolism, being a kind of "intracellular clock" that regulates the activities of the cell. ${ }^{5}$ Such an increase contributed to the life extension of both fibroblast cultures and the organism at large.

The expression of Clock and Perl genes in human skin cells, such as keratinocytes, melanocytes, and dermal fibroblasts, confirm the presence of circadian rhythms therein. $^{20}$ Circadian rhythms are adaptive mechanisms of live organisms, associated with the alternation of day and night, the main purpose of which is metabolism regulation. Studies have compared circadian rhythms in different types of skin cells and concluded that the skin is not only under the influence of the central suprachiasmatic nucleus clock but also possesses its own self-regulating circadian rhythms specific for each type of cells (keratinocytes, melanocytes, and fibroblasts). ${ }^{21}$

The CLOCK protein is involved in regulating the expression of $I C A M-1, V C A M-1$, and $C C L-2$ genes associated with cell adhesion. ${ }^{22}$ The effect of CLOCK protein deficiency on aging has been studied in mice. The functioning of circadian rhythm in the central clock did not stop, as the CLOCK transcription factor could be replaced with NPAS2, but such replacement is impossible in per-

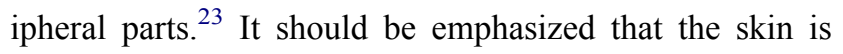
also a peripheral organ.

\section{Conclusions}

The obtained results indicate that treatment with hyaluronic acid facial filler influences the expression of anti-aging markers and thus they have a dermatoprotective effect. Cell culture tests show activation of synthetic activity, increased antioxidant properties of skin cells, and 
protection from premature aging of cells by the influence of HA fillers (Novacutan ${ }^{\circledR}$ ).

\section{Abbrevations}

HA-Y, Novacutan ${ }^{\circledR}$ YBio; HA-S, Novacutan ${ }^{\circledR}$ SBio.

\section{Disclosure}

The authors report no conflicts of interest in this manuscript.

\section{References}

1. Greta F. Plant complexity and cosmetic innovation. Iscience. 2020;23:101358.

2. Sani SHZ, Fathirezaie Z, Gerber M, et al. Self-esteem and symptoms of eating-disordered behavior among female adolescents. Psychol Rep. 2020;124:33294120948226.

3. Takaoka M, Okumura S, Seki T, Ohtani M. Effect of amino-acid intake on physical conditions and skin state: a randomized, doubleblind, placebo-controlled, crossover trial. J Clin Biochem Nutr. 2019;65(1):52-58. doi:10.3164/jcbn.18-108

4. Aguilar-Toalá JE, Hall FG, Urbizo-Reyes UC, et al. In silico prediction and in vitro assessment of multifunctional properties of postbiotics obtained from two probiotic bacteria. Probiotics Antimicrob Proteins. 2020;12:608-622.

5. Cai T, Hua B, Luo D, et al. The circadian protein CLOCK regulates cell metabolism via the mitochondrial carrier SLC25A10. Biochim Biophys Acta Mol Cell Res. 2019;1866(8):1310-1321. doi:10.1016/j. bbamcr.2019.03.016.

6. brandmonitor.ru [homepage on the Internet]. Online brand protection in Russia and the CIS. Moscow: Brand Monitor; [updated June 18, 2019; cited November 11, 2020]. Available from: https://brandmoni tor.ru/en/materials/obem-kontrafakta-na-rynke-esteticheskoy-medit siny-stremitelno-rastet/. Accessed April 7, 2021.

7. Fradette J, Godbout MJ, Michel M, Germain L. Localization of Merkel cells at hairless and hairy human skin sites using keratin 18 . Biochem Cell Biol. 1995;73(9-10):635-639. doi:10.1139/095-070

8. Xu Y, Sun Z. Molecular basis of Klotho: from gene to function in aging. Endocr Rev. 2015;36(2):174-193. doi:10.1210/er.2013-1079

9. Koyama D, Sato Y, Aizawa M, et al. Soluble $\alpha$ Klotho as a candidate for the biomarker of aging. Biochem Biophys Res Commun. 2015;467 (4):1019-1025. doi:10.1016/j.bbrc.2015.10.018

10. Kim JH, Hwang KH, Park KS, Kong ID, Cha SK. Biological role of anti-aging protein Klotho. J Lifestyle Med. 2015;5(1):1. doi:10.15280/jlm.2015.5.1.1
11. Sopjani M, Rinnerthaler M, Kruja J, Dërmaku-Sopjani M. Intracellular signaling of the aging suppressor protein Klotho. Curr Mol Med. 2015;15(1):27-37. doi:10.2174/ 1566524015666150114111258

12. Tan DX, Manchester LC, Esteban-Zubero E, Zhou Z, Reiter RJ. Melatonin as a potent and inducible endogenous antioxidant: synthesis and metabolism. Molecules. 2015;20(10):18886-18906. doi:10.3390/molecules201018886

13. Galano A, Tan DX, Reiter RJ. Melatonin: a versatile protector against oxidative DNA damage. Molecules. 2018;23(3):530. doi:10.3390/ molecules23030530

14. Dalio MB, Haikel Júnior LF, Dalio RB, et al. A study of the effects of pinealectomy on intestinal cell proliferation in infant newborn rats. Acta Cir Bras. 2006;21(1):16-20. doi:10.1590/S010286502006000100005

15. Tosini G, Owino S, Guillaume JL, Jockers R. Understanding melatonin receptor pharmacology: latest insights from mouse models, and their relevance to human disease. Bioessays. 2014;36(8):778-787. doi:10.1002/bies.201400017

16. Hess J, Angel P, Schorpp-Kistner M. AP-1 subunits: quarrel and harmony among siblings. J Cell Sci. 2004;117(25):5965-5973. doi:10.1242/jcs.01589

17. Garces de Los Fayos Alonso I, Liang HC, Turner SD, Lagger S, Merkel O, Kenner L. The role of activator protein-1 (AP-1) family members in CD30-positive lymphomas. Cancers. 2018;10(4):93. doi:10.3390/cancers 10040093

18. Qin Z, Robichaud P, He T, Fisher GJ, Voorhees JJ, Quan T. Oxidant exposure induces cysteine-rich protein 61 (CCN1) via c-Jun/AP-1 to reduce collagen expression in human dermal fibroblasts. PLoS One. 2014;9(12):115402. doi:10.1371/journal.pone.0115402

19. Yoshimura D, Sakumi K, Ohno M, et al. An oxidized purine nucleoside triphosphatase, MTH1, suppresses cell death caused by oxidative stress. J Biol Chen. 2003;278(39):37965-37973. doi:10.1074/jbc. M306201200

20. Zanello SB, Jackson DM, Holick MF. Expression of the circadian clock genes clock and period1 in human skin. J Invest Dermatol. 2000;115(4):757-760. doi:10.1046/j.1523-1747.2000.00121.x

21. Sandu C, Dumas M, Malan A, et al. Human skin keratinocytes, melanocytes, and fibroblasts contain distinct circadian clock machineries. Cell Mol Life Sci. 2012;69(19):3329-3339. doi:10.1007/ s00018-012-1026-1

22. Gao Y, Meng D, Sun N, et al. Clock upregulates intercellular adhesion molecule-1 expression and promotes mononuclear cells adhesion to endothelial cells. Biochem Biophys Res Commun. 2014;443 (2):586-591. doi:10.1016/j.bbrc.2013.12.022

23. DeBruyne JP, Weaver DR, Reppert SM. CLOCK and NPAS2 have overlapping roles in the suprachiasmatic circadian clock. Nat Neurosci. 2007;10(5):543-545. doi:10.1038/nn1884
Clinical, Cosmetic and Investigational Dermatology is an international, peer-reviewed, open access, online journal that focuses on the latest clinical and experimental research in all aspects of skin disease and cosmetic interventions. This journal is indexed on CAS.
The manuscript management system is completely online and includes a very quick and fair peer-review system, which is all easy to use. Visit http://www.dovepress.com/testimonials.php to read real quotes from published authors. 